\title{
Coupling of DEM and remote-sensing-based approaches for semi-automated detection of regional geostructural features in Zagros mountain, Iran.
}

\begin{abstract}
In recent years, remote-sensing data have increasingly been used for the interpretation of objects and mapping in various applications of engineering geology. Digital elevation model (DEM) is very useful for detection, delineation, and interpretation of geological and structural features. The use of image elements for interpretation is a common method to extract structural features. In this paper, linear features were extracted from the Landsat ETM satellite image and then DEM was used to enhance those objects using digital-imageprocessing filtering techniques. The extraction procedures of the linear objects are performed in a semi-automated way. Photographic elements and geotechnical elements are used as main keys to extract the information from the satellite image data. This paper emphasizes on the application of DEM and usage of various filtering techniques with different convolution kernel size applied on the DEM. Additionally, this paper discusses about the usefulness of DEM and satellite digital data for extraction of structural features in SW of Zagros mountain, Iran.
\end{abstract}

Keyword: Remote sensing; Image processing; DEM; Linear feature mapping; GIS; Zagros mountain 